Note

\title{
Electrochemical characterization of Biodiesel by linear voltammetry and electrochemical impedance spectroscopy
}

\author{
J. Villarreal-Castellon ${ }^{1,2}$, F.E. Mercader-Trejo ${ }^{2}$, A. Álvarez-López ${ }^{3}$, E.R. Larios-Durán ${ }^{4}$, \\ R. Antaño-López ${ }^{5}, R$. Herrera-Basurto ${ }^{6}, A$. Chacón-López ${ }^{1}, A$. Rodríguez-López ${ }^{2 *}$, U. López-García $^{1 *}$ \\ ${ }^{1}$ Tecnológico Nacional de México (TecNM)-Instituto Tecnológico de Tepic-Laboratorio de \\ Biotecnología, Av. Tecnológico \# 2595, Col. Lagos del Country, C.P. 63175, Tepic, Nayarit, México. \\ ${ }^{2}$ Universidad Politécnica de Santa Rosa Jáuregui (UPSRJ), km 31+150, carretera federal 57 tramo \\ Qro-SLP, Parque Industrial Querétaro, Santa Rosa Jáuregui, C.P.76220 Querétaro, México. \\ ${ }^{3}$ Universidad Tecnológica de Corregidora. Carretera Santa Bárbara-Coroneo, Km. 11.2. Corregidora, \\ Querétaro, México. \\ ${ }^{4}$ Universidad de Guadalajara, Departamento de Ingeniería Química, Blvd. Marcelino García Barragán \\ \#1451, C.P. 44430, Guadalajara Jalisco, México. \\ ${ }^{5}$ Centro de Investigación y Desarrollo Tecnológico en Electroquímica, S.C. Parque Tecnológico \\ Querétaro s/n, Sanfandila, Pedro Escobedo, Querétaro, México. \\ ${ }^{6}$ Universidad Aeronáutica en Querétaro. Carretera Estatal 200 Querétaro Tequisquiapan No. 22154 \\ C.P. 76270, Colón, Querétaro. México. \\ *E-mail: arodriguez@upsrj.edu.mx, ulopez@ittepic.edu.mx
}

doi: $10.20964 / 2018.06 .59$

Received: 8 September 2017 / Accepted: 25 January 2018 / Published: 10 May 2018

Biodiesel from different sources were synthetized according to transesterification method. Actually, there are different methods to characterize these fuels, but majority are physicochemical techniques, some of they, like water content by Karl-Fischer method are really complex. Because electrochemical techniques offer fast and accurate results, an electrochemical cell was designed and built to measure these biodiesels, in particular electrolytic conductivity, which is an important parameter that characterizes solutions in different fields of research and industry. Biodiesel obtained from castor oil present the lowest electrolytic conductivity from four evaluated.

Keywords: Biodiesel, Electrochemical Characterization, Linear Voltammetry, Electrochemical Impedance Spectroscopy. 
(C) 2018 The Authors. Published by ESG (www.electrochemsci.org). This article is an open access article distributed under the terms and conditions of the Creative Commons Attribution license (http://creativecommons.org/licenses/by/4.0/). 